\title{
México: la combinación de las predicciones mensuales de inflación mediante encuestas
}

\author{
Pilar Poncela, Victor M. Guerrero, Alejandro Islas, \\ Julio Rodríguez y Rocio Sánchez-Mangas
}

RESUMEN

Se examinan las combinaciones de las predicciones o proyecciones inflacionarias en México mediante encuestas quincenales a expertos. La inflación de los precios al consumidor se mide dos veces cada mes empleando varios métodos de combinación. Se aconseja usar técnicas reductivas de la dimensión, comparables con diferentes métodos de referencia, incluida la predicción más sencilla basada en el promedio. La imputación de los valores faltantes de la base de datos se realiza mediante dos métodos diferentes, cuyos resultados son básicamente robustos a la elección del método. El análisis preliminar se basó en la estructura de datos de panel y mostró la posible utilidad de emplear técnicas de reducción de la dimensión para combinar las predicciones de los expertos. Las principales conclusiones son: las primeras proyecciones mensuales se combinan mejor mediante el primer componente principal de las predicciones disponibles; la mejor segunda se obtiene calculando la proyección mediana y es más precisa.

PALABRAS CLAVE

CLASIFICACIÓN JEL

AUTORES
Inflación, proyecciones económicas, México

E37, E53

Pilar Poncela es profesora titular del Departamento de Economía Cuantitativa de la Universidad Autónoma de Madrid, España. pilar.poncela@uam.es

Víctor M. Guerrero es profesor de tiempo completo en el Departamento Académico de Estadística del Instituto Tecnológico Autónomo de México (IтAM), México.guerrero@itam.mx

Alejandro Islas es profesor de tiempo completo en el Departamento Académico de Estadística del Instituto Tecnológico Autónomo de México (IтAM), México. aislas@itam.mx

Julio Rodríguez es profesor titular del Departamento de Economía Cuantitativa de la Universidad Autónoma de Madrid, España.jr.puerta@uam.es

Rocío Sánchez-Mangas es profesora titular del Departamento de Economía Cuantitativa de la Universidad Autónoma de Madrid, España. rocio.sanchez@uam.es 


\section{I}

\section{Introducción}

Al decir de todos, con la política monetaria de México de los últimos 25 años se ha logrado alcanzar exitosamente la estabilidad de los precios: la tasa de inflación media disminuyó del $4,3 \%$ por mes en los años ochenta al $0,4 \%$ mensual en los primeros años del siglo XXI. Para asegurar la estabilidad de los precios, las autoridades mexicanas utilizaron diferentes instrumentos monetarios, que van desde el control del tipo de cambio o de la base monetaria al establecimiento de metas de inflación. A fines de la década de 1980, durante un período caracterizado por una marcada inestabilidad macroeconómica, las autoridades monetarias decidieron producir un índice de inflación quincenal. El objetivo de dicha periodicidad era difundir información más oportuna sobre las dinámicas de precios durante los períodos de volatilidad, de modo que los agentes económicos, públicos y privados, pudieran seguir de cerca la evolución de los precios en la economía para tomar decisiones que les permitieran optimizar el uso de sus recursos.

Las predicciones de inflación frecuentes son importantes, tanto para los operadores de mercado como para los institucionales. Por una parte, los operadores del mercado financiero tienden a renovar sus expectativas continuamente a medida que se divulga información actualizada, y a utilizar esa información para modificar sus estrategias de inversión. Por otra parte, de acuerdo con Woodford (2003), la revisión oportuna de las proyecciones macroeconómicas es fundamental para conducir la política monetaria sobre la base de las expectativas del mercado. La precisión y la oportunidad de las proyecciones de inflación a corto plazo pueden influir considerablemente en esas estrategias.

El objetivo de este trabajo consiste en producir una combinación eficaz de predicciones de inflación para México. El marco de las proyecciones se basa en las técnicas de reducción de la dimensión propuestas

Este trabajo contó con el apoyo financiero de un Proyecto de cooperación interuniversitaria UAM-BANCO SANTANDER con América Latina. La Asociación Mexicana de Cultura colaboró para la participación de V. M. Guerrero y A. Islas en este proyecto. Los autores desean agradecer a Joel Virgen del Banco Nacional de México (BANAMEX) por proporcionar las bases de datos y responder a las preguntas sobre aspectos complejos de los datos. P. Poncela, J. Rodríguez y R. Sánchez-Mangas agradecen el apoyo financiero recibido en el marco del proyecto ECO2012-32854 del Gobierno de España. por Poncela y otros (2011), que permiten obtener una única predicción de inflación más precisa en lugar de varias predicciones individuales. Se utilizan técnicas de reducción de la dimensión para extraer la información común contenida en las proyecciones de los expertos, producir una proyección consensual y revelar el nivel de desacuerdo entre los diferentes analistas.

Como es sabido, la combinación de predicciones aumenta la exactitud de la predicción al aprovechar la disponibilidad de información de múltiples fuentes. Desde el artículo seminal de Bates y Granger (1969) se han desarrollado muchos métodos de combinación, que van desde el simple promedio a las alternativas más recientes, como las técnicas de reducción de la dimensión (véase más información sobre este tema en Aiolfi, Capistrán y Timmermann (2011); Timmermann (2006), y Newbold y Harvey (2002), entre otros).

En México existen dos encuestas a analistas profesionales, realizadas respectivamente por el banco central de México y el Banco Nacional de México (BANAMEX) (el segundo mayor banco privado del país). Debido a que la encuesta del banco central no está disponible públicamente, las predicciones de la inflación mexicana se basan en los datos proporcionados por BANAMEX dos veces cada mes desde 2007. Dicha encuesta ofrece proyecciones regulares de variables macroeconómicas relacionadas con la inversión y la producción. En el presente trabajo solo se tienen en cuenta las predicciones de la inflación mensual de 2007 a 2011 con un período de antelación. Después de divulgada la proyección de cada mes, esta se revisa en virtud de la nueva información disponible entre una encuesta y otra, de manera de ofrecer dos predicciones para el mismo mes. Al haber pocas observaciones puede surgir el llamado forecast combination puzzle, por el hecho de que la media de las predicciones de la muestra produzca mejores resultados de proyección que los esquemas de ponderación más sofisticados (véanse, por ejemplo, Smith y Wallis (2009), y Aiolfi, Capistrán y Timmermann (2011). En este estudio se procura sugerir procedimientos de combinación de proyecciones que puedan funcionar mejor cuando la muestra es bastante breve. Se asume también que la segunda predicción es más acertada que la primera, porque incluye la información sobre la inflación registrada en la primera 
quincena del mes. En consecuencia, no es necesario utilizar métodos de frecuencia mixtos como el Mixed Data Sampling (MIDAs) (véase, por ejemplo, Ghysels, Santa-Clara y Valkanov, 2004), en los que se podrían combinar predicciones más frecuentes (en este caso quincenales), pues la proyección de inflación relativa a la primera quincena del mes puede reemplazarse por su medida real. En la sección II se explican en detalle los datos proporcionados por la encuesta de analistas profesionales.

Este trabajo se relaciona con otros. Por ejemplo, Poncela y Senra (2006) emplearon dos componentes principales para combinar las predicciones de inflación de los Estados Unidos de América y relacionaron el segundo componente con el nivel de inflación esperado. Los trabajos de Capistrán y López-Moctezuma (2010a y 2010b) también se refieren a la predicción de la inflación mexicana, pero esos autores utilizaron la encuesta mensual realizada por el banco central de México y su objetivo era muy distinto al de este estudio. En efecto, mientras que en su primer trabajo procuran mostrar que la proyección consensuada de un conjunto de variables macroeconómicas, entre ellas la inflación, no pasa las pruebas de imparcialidad, falta de correlación serial y uso eficiente de la información disponible, en el segundo estudian la medida en que la información de que disponen los analistas se incorpora en forma eficiente a las predicciones de inflación y crecimiento del producto interno bruto (PIB).

Este trabajo se divide en cinco secciones, incluida esta Introducción. En la segunda sección se presentan la notación utilizada en el estudio y la información relativa al tratamiento de los datos faltantes, así como la estructura de panel de la encuesta formulada a los analistas profesionales. En la tercera sección se describen las técnicas de reducción de la dimensión utilizadas para producir una única predicción, mientras que en la cuarta sección se presentan los resultados de la aplicación de esas técnicas a los datos mexicanos. En la quinta y última sección se presentan las conclusiones alcanzadas.

\section{II}

\section{Notación y preparación de los datos}

En los cálculos siguientes, $p_{\tau}$ denota el porcentaje de inflación quincenal mediante la variación del índice de precios al consumidor (IPC) quincenal ( $q I P C$ ) para $\tau=2 t-1,2 t$, donde $t=1, \ldots, T$ expresa los meses, es decir, $p_{\tau}=100\left(\mathrm{qIPC}_{\tau}-\mathrm{qIPC}_{\tau-1}\right) / \mathrm{qIPC}_{\tau-1}$, mientras que el porcentaje de inflación mensual, $\pi_{\mathrm{t}}=100\left(\mathrm{IPC}_{\mathrm{t}}-\mathrm{IPC}_{\mathrm{t}-1}\right) /$ $\mathrm{IPC}_{\mathrm{t}-1}$, se basa en la variación mensual del IPC dada por $\mathrm{IPC}_{\mathrm{t}}=\left(\mathrm{qIPC}_{2 \mathrm{t}-1} \mathrm{IPC}\right) / 2$. Las cifras oficiales relativas a la inflación son divulgadas por el Instituto Nacional de Estadística, responsable de calcular el IPC el día 9 de cada mes con respecto al mes anterior y el día 24 con respecto a la primera quincena del mes. Las cifras oficiales de $\pi_{\mathrm{t}} \mathrm{y} p_{\tau}$ están disponibles en línea en el sitio web www.inegi.org.mx.

Si bien la encuesta ofrece predicciones de distintas variables macroeconómicas, este trabajo se concentra en las proyecciones del porcentaje de inflación realizadas por cada uno de los $i=1, \ldots, N$ expertos: la predicción de inflación para la primera quincena del mes, $y_{i, \tau \mid \tau-1}, \mathrm{y}$ dos predicciones de inflación, $z_{i, t}$, que difieren según el momento en que se obtienen y la información empleada por los analistas. Para destacar esta diferencia, la notación utilizada para las proyecciones de inflación mensuales $z_{i, t \uparrow \tau}$ es la siguiente: el primer subíndice $t$ se mide en unidades mensuales, mientras que el segundo, $\tau$, se mide quincenalmente. Las tres predicciones de inflación se obtienen de la siguiente manera:

i) alrededor del día 20 de cada mes (tres o cuatro días antes de la publicación de la cifra correspondiente a la primera quincena del mes) los expertos realizan una proyección de la inflación para la primera quincena de ese mes. Por consiguiente, los expertos disponen de la información hasta la segunda quincena del mes anterior y dicha predicción se denota como $y_{i, 2 \mathrm{t}-1 \mid 2(\mathrm{t}-1)}$. Al mismo tiempo, los expertos prevén la inflación mensual para el mes en curso, que se denomina proyección mensual 1 y se denota como $z_{i, t \mid 2(\mathrm{t}-1)}$. A continuación, ii) alrededor del día 6 de cada mes (una vez más tres o cuatro días antes de la publicación de la cifra oficial) los expertos realizan otra predicción de inflación mensual relativa al mes anterior, denominada proyección mensual 2, y expresada como $z_{i, t \mid 2 t-1}$.

Las predicciones, ya sea relativas a la primera quincena o a todo el mes, están siempre condicionadas a la información concerniente a la quincena anterior. El esquema de generación de proyecciones se puede apreciar en el cuadro 1. De ese modo, la encuesta de analistas profesionales proporciona predicciones con 


\begin{tabular}{|c|c|c|c|c|c|}
\hline \multicolumn{2}{|c|}{ Período } & \multicolumn{3}{|c|}{ Predicciones del porcentaje de inflación } & \multirow{2}{*}{$\begin{array}{c}\text { Predicción realizada } \\
\text { el día... }\end{array}$} \\
\hline Mes & Quincena & Primera quincena & & & \\
\hline $\mathrm{T}$ & $\tau$ & $y_{i, 2 t-1 \mid 2(t-1)}$ & $z_{i, t \mid 2(t-1)}$ & $z_{i, t \mid 2 t-1}$ & \\
\hline \multirow[t]{2}{*}{1} & 1 & $y_{i, 1 \mid 0}$ & $z_{i, 1 \mid 0}$ & - & 20 del mes 1 \\
\hline & 2 & - & - & $z_{i, 1 \mid 1}$ & 6 del mes 2 \\
\hline \multirow[t]{2}{*}{2} & 3 & $y_{i, 3 \mid 2}$ & $z_{i, 2 \mid 2}$ & - & 20 del mes 2 \\
\hline & 4 & - & - & $z_{i, 2 \mid 3}$ & 6 del mes 3 \\
\hline$\cdots$ & $\cdots$ & $\cdots$ & $\cdots$ & $\cdots$ & $\ldots$ \\
\hline \multirow[t]{2}{*}{$\mathrm{T}$} & $2 \mathrm{~T}-1$ & $y_{i, 2 T-1 \mid 2(T-1)}$ & $z_{i, T \mid 2(T-1)}$ & - & 20 del mes $\mathrm{T}$ \\
\hline & $2 \mathrm{~T}$ & - & - & $z_{i, T \mid 2 T-1}$ & 6 del mes $T+1$ \\
\hline
\end{tabular}

Fuente: elaboración propia.

relación a la primera quincena de cada mes, $y_{i, 2 \mathrm{t}-1 \mid 2(\mathrm{t}-1)}$, y proyecciones mensuales $z_{i, t \mid 2(\mathrm{t}-1)}$ y $z_{i, t \mid 2 \mathrm{t}-1}$ para los meses $t=1, \ldots, T$, con $T=60$ (período de enero de 2007 a diciembre de 2011) y $N=18$ especialistas. $\mathrm{Si}$ bien el número de analistas que participan en la encuesta ha cambiado con los años, hubo aproximadamente 18 encuestados regulares en cada encuesta (se excluye de este estudio a los expertos que abandonaron el grupo y a los que ingresaron recientemente).

Debido a que en la base de datos de la encuesta original faltan algunos valores correspondientes a todos los expertos en distintas fechas, se decidió utilizar un procedimiento de estimación sistemática con objeto de cubrir esas lagunas. Para verificar la sensibilidad de los resultados se propusieron dos procedimientos diferentes, cada uno de los cuales se seleccionó con los siguientes criterios: i) que hiciera uso solo del registro histórico de predicciones del experto de referencia, y ii) que tuviera en cuenta algunas características principales de los datos observados. Si bien el procedimiento de estimación de datos faltantes incluido en el programa Time Series Regression with ARIMA Noise, Missing Observations and Outliers (TRAMO) (véase Gómez y Maravall, 1996), disponible en línea en el sitio web del Banco de España, es óptimo y fácil de usar, su aplicación fue descartada porque no cumple con el mencionado criterio i). El primer procedimiento utilizado se basa en la falta de una tendencia observable en la serie de predicciones de todos los expertos, como puede apreciarse en el gráfico 1 relativo al caso de un analista en particular (BANAMEX). En ese contexto, las primeras diferencias de la serie se pueden considerar razonablemente constantes en el tiempo, de modo que el promedio de las diferencias pasadas proporciona una estimación razonable de la diferencia actual con respecto a la predicción correspondiente, ya sea mensual o quincenal. En consecuencia, para la primera proyección mensual de todo el mes se tiene

$$
\begin{gathered}
z_{i, t \mid 2(t-1)}-z_{i, t-1 \mid 2(t-1)-2}= \\
\sum_{j=1}^{t-2} \frac{z_{i, t-j \mid 2(t-j)-2}-z_{i, t-1-j \mid 2(t-j-1)-2}}{t-2}
\end{gathered}
$$

de manera que la predicción correspondiente al mes t, considerados los datos disponibles hasta el momento $2(t-1)$, está dada por

$z_{i, t \mid 2(t-1)}=z_{i, t-1 \mid 2(t-1)-2}+\frac{\left(z_{i, t-1 \mid 2(t-1)-2}-z_{i, 1 \mid 0}\right)}{(t-2)}(2)$

y para la segunda predicción mensual se obtiene

$z_{i, t \mid 2 t-1}=z_{i, t-1 \mid 2(t-1)-1}+\frac{\left(z_{i, t-1 \mid 2(t-1)-1}-z_{i, 1 \mid 1}\right)}{(t-2)}$

En forma análoga, para la primera proyección quincenal se tiene

$$
\begin{gathered}
y_{i, 2 t-1 \mid 2(t-1)}-y_{i, 2(t-1) \mid 2(t-1)-1}= \\
\sum_{j=1}^{2(t-1)-1} \frac{y_{i, 2 t-1-j \mid 2(t-1)-j}-y_{i, 2(t-1)-j \mid 2(t-1)-1-j}}{2(t-1)-1}
\end{gathered}
$$

que lleva a

$$
\begin{gathered}
y_{i, 2 t-1 \mid 2(t-1)}=y_{i, 2(t-1) \mid 2(t-1)-1}+ \\
\frac{\left(y_{i, 2(t-1) \mid 2(t-1)-1}-y_{i, 1 \mid 0}\right)}{2(t-1)-1}
\end{gathered}
$$


El segundo procedimiento surge de la inspección de la estructura de autocorrelación de las cifras de inflación oficiales. Allí se observa que se necesita una diferencia estacional de orden 12 para que la serie sea aproximadamente estacionaria. A continuación, visto que las predicciones tratan de parecerse a las cifras oficiales, se supone que las series de proyecciones de todos los expertos comparten el mismo orden de integración. Por lo tanto, se utilizan las siguientes expresiones para estimar los valores faltantes de la primera y segunda predicciones mensuales

$$
\begin{gathered}
z_{i, t \mid 2(t-1)}=z_{i, t-12 \mid 2 t-26} \\
+\sum_{j=1}^{t-13} \frac{\left(z_{i, t-j \mid 2(t-j)-2}-z_{i, t-12-j \mid 2(t-j)-26}\right)}{(t-13)}
\end{gathered}
$$

$\mathrm{y}$

$$
\begin{gathered}
z_{i, t \mid 2 t-1}=z_{i, t-12 \mid 2 t-25} \\
+\sum_{j=1}^{t-13} \frac{\left(z_{i, t-j \mid 2(t-j)-1}-z_{i, t-12-j \mid 2(t-j)-25}\right)}{(t-13)}
\end{gathered}
$$

que son válidas para $t=14, \ldots, T$. De manera análoga, la estimación de una predicción faltante para la primera quincena del mes está dada por

$$
\begin{gathered}
y_{i, 2 t-1 \mid 2(t-1)}=y_{i, 2 t-13 \mid 2 t-14}+ \\
\sum_{j=1}^{t-7} \frac{\left(y_{i, 2(t-j)-1 \mid 2(t-j-1)}-y_{i, 2(t-j)-13 \mid 2(t-j)-14}\right)}{(t-7)}
\end{gathered}
$$

para $t=7, \ldots, T$, con la suma igual a 0 si $t=7$. En caso de no poderse calcular las expresiones anteriores (porque $t<7$ ), los valores faltantes se reemplazaron con las cifras oficiales. En el gráfico 1 puede verse un ejemplo de la aplicación del método 2, con el que se estimaron los siguientes valores faltantes: las primeras predicciones mensuales de diciembre de 2007 y octubre de 2011; las segundas proyecciones mensuales de abril de 2008, diciembre de 2008, diciembre de 2009 y diciembre de 2010; y las primeras predicciones quincenales de diciembre de 2007 y octubre de 2011. De hecho, puede decirse que faltaban las observaciones de todos los expertos porque, por distintas razones, la encuesta no fue enviada en esas fechas. En consecuencia, estrictamente hablando, no se trata de valores faltantes.

Es interesante señalar que las predicciones de inflación con respecto a la segunda quincena de cada mes, $y_{i, 2 t \mid 2 \mathrm{t}-1}$, pueden derivarse de las proyecciones mensuales $z_{i, t \mid 2 \mathrm{t}-1}$, dado que en el momento en que se realizan, la cifra de la inflación quincenal oficial de la primera quincena del mes, $p_{2 t-1}$, ya está disponible. En el

GRÁFICO 1

Primeras y segundas predicciones mensuales (mensual 1 y mensual 2) y predicciones para la primera quincena del mes (En porcentajes)

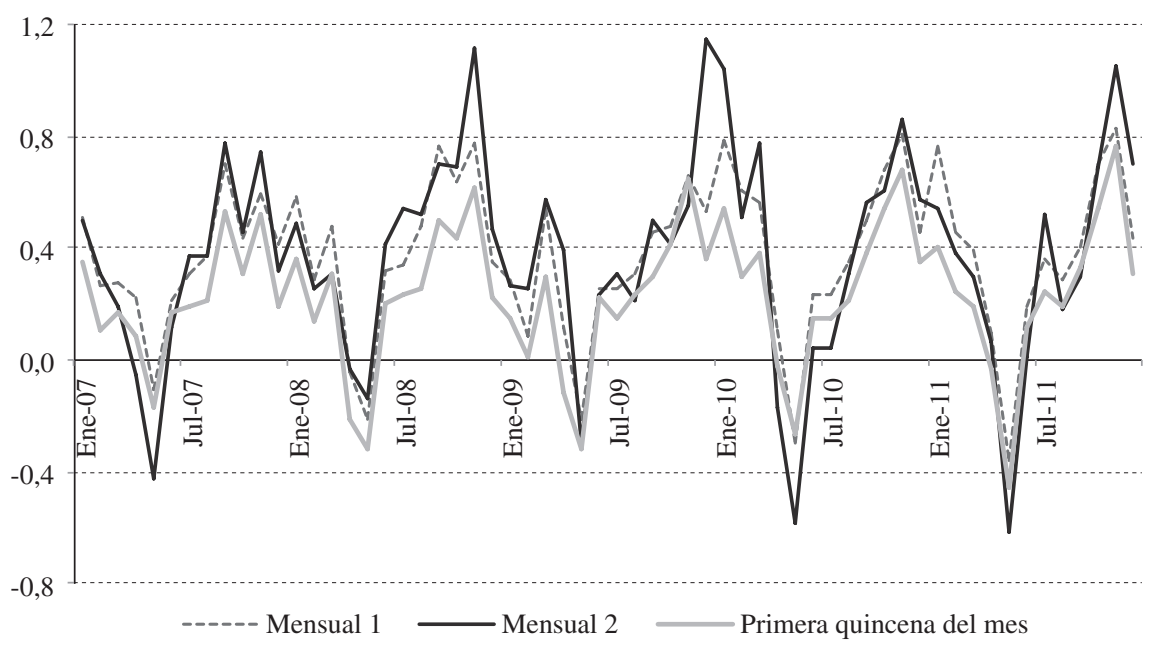

Fuente: elaboración propia. 
apéndice se muestra cómo se derivan estas predicciones. No obstante, se señala que las proyecciones atinentes a la segunda quincena de cada mes no agregan realmente más información a la que se detalla en el cuadro 1, pues se derivan de la segunda predicción mensual.

\section{Análisis con datos de panel}

Como se mencionó anteriormente, los datos de la encuesta de analistas profesionales utilizados en este trabajo tienen una estructura de panel, con 18 unidades individuales - los expertos - y 60 períodos de tiempo, es decir, sus predicciones mensuales (o relativas a la primera quincena del mes) desde enero de 2007 a diciembre de 2011. Se puede aprovechar la estructura de datos de panel para descomponer la proyección realizada por el experto $i$ en el momento $t$ en varios componentes. A partir de las proyecciones mensuales, se puede escribir

$$
z_{i, t \mid \tau}=z_{i}+z_{t}+\varepsilon_{i, t \mid \tau}
$$

donde $\tau=2(t-1)$ para las primeras predicciones mensuales y $\tau=2 t-1$ para las segundas.

El primer componente, $z_{i}$, representa el efecto individual invariante en el tiempo. Este captura las características intrínsecas del experto $i$ y puede escribirse de la siguiente manera

$$
z_{i}=\frac{1}{T} \sum_{t=1}^{T} z_{i, t \mid \tau}
$$

para $i=1, \ldots, N$. Captura el nivel medio de las predicciones realizadas por el analista $i$ en el período de la muestra. El segundo componente, $z_{t}$, es un efecto agregado que no varía de un individuo a otro y capta la dinámica común de las proyecciones realizadas por los expertos. Se puede escribir como

$$
z_{t}=\frac{1}{N} \sum_{i=1}^{N}\left(z_{i, t \mid \tau}-z_{i}\right)
$$

para $t=1, \ldots, T$. Este componente calcula la media de las predicciones realizadas por todos los expertos con respecto al período $t$, una vez eliminados los efectos individuales. El tercer componente es un término de error dado por $\varepsilon_{i, t \mid \tau}=z_{i, t} \mid \tau-z_{i}-z_{t}$, que presenta tanto variación temporal como individual, y corresponde a la parte de la predicción que no puede aislarse ni como efecto invariante en el tiempo ni como efecto invariante de un individuo a otro.

El efecto individual (invariante en el tiempo) y el efecto agregado (invariante de un individuo a otro) son ortogonales por construcción. El término de error es el residuo de la proyección de $z_{i, t \mid \tau}$ en esos componentes, y por lo tanto, es ortogonal a ellos. La ortogonalidad de los componentes permite que la varianza de la predicción $z_{i, t} \tau$ se escriba como la suma de la varianza de cada componente. Esta descomposición brinda información sobre el aporte de los efectos específicos de cada individuo y las creencias comunes de los expertos a la varianza total. La descomposición de panel también puede aplicarse a los errores de las predicciones, con una interpretación análoga de los componentes. Teniendo en cuenta los patrones de las proyecciones, se aplicó esta descomposición a los errores de proyección. Los resultados se detallan en el cuadro 2 . Allí se muestra la varianza total de los errores de predicción, para los dos métodos de imputación utilizados, con respecto a las primeras predicciones mensuales (columnas 1 y 2

\begin{tabular}{|c|c|c|c|c|}
\hline & \multicolumn{4}{|c|}{ Error de predicción } \\
\hline & \multicolumn{2}{|c|}{$\begin{array}{l}\text { Primera predicción mensual } \\
\qquad \pi_{t}-z_{i, t} \mid 2(t-1)\end{array}$} & \multicolumn{2}{|c|}{$\begin{array}{l}\text { Segunda predicción mensual } \\
\qquad \pi_{t}-z_{i, t} \mid 2 t-1\end{array}$} \\
\hline & $\begin{array}{l}\text { Método de } \\
\text { imputación } 1\end{array}$ & $\begin{array}{l}\text { Método de } \\
\text { imputación } 2\end{array}$ & $\begin{array}{l}\text { Método de } \\
\text { imputación } 1\end{array}$ & $\begin{array}{l}\text { Método de } \\
\text { imputación } 2\end{array}$ \\
\hline Varianza total & 0,044 & 0,042 & 0,025 & 0,026 \\
\hline \multicolumn{5}{|c|}{ Contribución a la varianza total del error de predicción (en porcentajes) } \\
\hline $\begin{array}{l}\text { Efecto individual } \\
\text { Efecto agregado } \\
\text { Término residual }\end{array}$ & $\begin{array}{r}1,08 \\
77,29 \\
21,63\end{array}$ & $\begin{array}{r}1,12 \\
78,21 \\
20,67\end{array}$ & $\begin{array}{r}2,54 \\
55,52 \\
41,94\end{array}$ & $\begin{array}{r}0,55 \\
74,35 \\
25,10\end{array}$ \\
\hline
\end{tabular}

Descomposición de panel de la varianza de los errores de predicción

Fuente: elaboración propia. 
del cuadro 2) y a las segundas proyecciones mensuales (columnas 3 y 4 del cuadro 2). También se muestra el aporte porcentual a esa varianza de los componentes individual, agregado y residual.

Como se esperaba, la varianza de los errores de proyección es menor en la segunda predicción mensual que en la primera, pues los expertos cuentan con más información en el momento de formularla. Con respecto a la descomposición de panel, es evidente que los efectos individuales no explican en gran medida la varianza total de los errores de proyección. El componente más importante es el efecto agregado. La característica principal de esos resultados es la información que proporcionan sobre la posible utilidad de los diferentes esquemas de combinación de predicciones. En el caso de la segunda predicción mensual, el aporte del efecto agregado a la varianza del error de proyección es menor que en la primera. En términos de la varianza de la predicción, esto significa que la contribución del efecto agregado es mayor en la segunda proyección mensual que en la primera. Por lo tanto, la variabilidad de las proyecciones en el segundo caso proviene casi totalmente de las creencias comunes de los expertos, representados por el efecto agregado. Sería de esperar que los esquemas de combinación sencillos, como el promedio o la mediana, funcionaran bien. En las primeras predicciones mensuales, el aporte del efecto agregado es mayor en el error de predicción (es decir, menor en la proyección) y por ende puede que los esquemas de combinación más sofisticados, como las técnicas de reducción de la dimensión que se muestran más adelante, funcionen mejor que los métodos más sencillos.

\section{III}

\section{Resumen de las técnicas de reducción de la dimensión}

Las técnicas de reducción de la dimensión para la combinación de predicciones fueron introducidas por Poncela y Senra (2006) y posteriormente ampliadas por Poncela y otros (2011). La idea clave consiste en considerar la combinación de proyecciones como una manera de reducir la dimensión de $N$ (el número de analistas en cada período de tiempo) a uno. Esto puede hacerse en dos etapas: en primera instancia, se debe reducir el número de predicciones individuales a solo $r \geq 1$ combinaciones lineales de ellas. Cada combinación lineal se forma de la siguiente manera

$$
f_{j s}=w_{j s}{ }^{\prime} x_{s}, \quad j=1, \ldots, r
$$

donde $w_{j s}$ es el vector de ponderación para la j-ésima combinación lineal respecto del período de predicción $s, \mathrm{y} x_{s}=\left(x_{1, s}, \ldots, \mathrm{x}_{N, s}\right)^{\prime}$ es $N$-vector de proyecciones por período de tiempo $s$ con cualquiera de los tres posibles tipos de predicciones disponibles en la encuesta. En otras palabras, $x_{i, s}$ podría ser igual a $y_{i, 2 t-1 \mid 2(t-1)}$ si se trabaja con proyecciones realizadas para la primera quincena del mes con datos hasta el mes anterior; $z_{i, t \mid 2(t-1)}$ si se utilizan las predicciones mensuales en $t$ con datos hasta el mes anterior; o $z_{i, t \mid 2 t-1}$ en el caso de la predicción quincenal previa del mes en curso.

En una segunda etapa se debe hacer una regresión de las combinaciones lineales sobre datos ya conocidos del tipo que se ha de prever, a la que se puede agregar un intercepto para la corrección del sesgo. En este caso solo se utilizó una combinación lineal, $r=1$, pues hay una gran similitud entre todos los analistas (todos ellos tratan de prever la inflación en un determinado período), y eso es lo que se trata de captar mediante las técnicas de reducción de la dimensión. Por otra parte, esta opción también se verificó empíricamente mediante un análisis con hasta tres componentes, cuyo objetivo era determinar la opción que proporcionaba la predicción con el menor error cuadrático medio (Root Mean Square Error-RMSE). Entonces,

$$
\pi_{s-1}=\beta_{0}+\beta_{1} f_{s-1}+e_{s-1}
$$

donde los coeficientes se estiman mediante mínimos cuadrados ordinarios (MCO), con datos observados hasta el período $s-1$ de manera de generar una verdadera proyección ex ante relativa al período $s$. Cuando la 
variable que se ha de prever y sus predicciones no son estacionarias se deben cointegrar, como se subrayó al presentar el método de imputación 2.

Las técnicas de reducción de la dimensión utilizadas en la primera etapa del procedimiento son las siguientes: componentes principales, modelos de factores estáticos y dinámicos, y mínimos cuadrados parciales. La principal diferencia entre el análisis de componentes principales y la técnica de mínimos cuadrados parciales es que mientras que en el primero no se tiene en cuenta la variable que se ha de prever al reducir la dimensión del problema para formar la combinación lineal, en la última sí se hace. A continuación, se presenta una breve reseña de estos métodos.

\section{Componentes principales}

Sea $z_{s}$ un $N \times 1$ vector de variables aleatorias de modo que $\operatorname{var}\left(z_{s}\right)=S$ para todos $s=1,2, \ldots, T$. El primer componente principal se define como la combinación lineal dada por el vector de ponderación $w=\left(w_{1}, \ldots, w_{N}\right)$, de modo que $w$ es el maximizador de $w$ ' $S w$ sujeto a $w^{\prime} w=1$. Lee y Carter (1992) propusieron un componente principal no estacionario.

\section{Modelos de factores}

Poncela y otros (2011) demostraron que los modelos de factores simples son más adecuados para la combinación de predicciones que los esquemas de factores más complejos, probablemente porque el número de parámetros de ponderación estimados para la combinación es menor. En particular, esos autores hallaron que los modelos de factores estáticos funcionaban bastante bien. Cuando hay un factor, el vector $x_{s}$ se descompone como la suma de dos componentes ortogonales: un factor común $f_{\mathrm{s}}$ más un error idiosincrásico $\eta_{s}$, como

$$
x_{s}=P f_{s}+\eta_{s}
$$

donde $P$ es la matriz de carga del factor $(N \times 1)$ y $Q=$ $\operatorname{var}\left(\eta_{s}\right)$ es una matriz diagonal.

En los modelos de factores dinámicos, tanto los componentes comunes como los idiosincrásicos pueden mostrar un comportamiento dinámico. Se asumen procesos autorregresivos para ambos tipos de componentes. En otras palabras, la ecuación para el factor común es

$$
\phi(B) f_{s}=u_{s}
$$

donde $\phi(B)=1-\phi_{1} B-\ldots-\phi_{p} B^{p} \operatorname{con} B$ el operador de rezago, $p<\infty$ y el error $u_{s}$ proviene de un proceso de ruido blanco. La ecuación para los componentes idiosincrásicos es

$$
\Phi(B) \eta_{s}=v_{s}
$$

donde $\Phi(B)=\mathrm{I}-\Phi_{1} B-\ldots-\Phi_{q} B^{q}$ es una matriz polinómica diagonal con $q<\infty$ y $v_{s}$ proviene de un proceso de ruido blanco multivariado con matriz de varianza diagonal $R$ $=\operatorname{var}\left(v_{s}\right)$. Si en los modelos de factores dinámicos el componente idiosincrásico es ruido blanco, el modelo corresponde al tipo indicado en Peña y Box (1987). En ese caso, la estructura de varianza-covarianza de los datos es

$$
\begin{gathered}
C(k)=E\left(x_{s}-\mu_{x}\right)\left(x_{s-k}-\mu_{x}\right)^{\prime}= \\
E\left(f_{s}-\mu_{f}\right)\left(f_{s-k}-\mu_{f}\right) P P^{\prime}
\end{gathered}
$$

donde $\mu_{x}=E\left(x_{s}\right)$ y $\mu_{f}=E\left(f_{s}\right)$. A continuación, el vector de carga de factor $P$ se asocia con el valor propio distinto de cero de las matrices de covarianza rezagadas y es el mismo para todos los intervalos distintos de cero. Si los componentes idiosincrásicos no corresponden a ruido blanco, la descomposición anterior es solo aproximada. En el ejercicio de predicción, este tipo de modelos de factores se denota como L1FM. Peña y Poncela (2004 y 2006) y Lam, Yao y Bathia (2011) extendieron este modelo al caso no estacionario, mientras que Alonso y otros (2011) analizaron los modelos de factores dinámicos estacionales.

\section{Mínimos cuadrados parciales}

El primer componente de mínimos cuadrados parciales se construye proyectando cada predicción en la dirección de la variable observada (en este caso, la inflación). El objetivo es tener explícitamente en cuenta la variable objeto de la proyección al formar la predicción común. De hecho, el análisis de regresión de mínimos cuadrados parciales supone que tanto las variables $X$ (en este caso, las predicciones de inflación) como la variable de respuesta $Y$ (es decir, la variable objeto de la proyección) dependen de variables latentes que están relacionadas. Se recuerda que $x_{s}$ es el $N$-vector de proyecciones para el período $s$ y la respuesta es la inflación registrada $\pi_{s}$. Entonces,

$$
x_{s}=P l_{s}+u_{s}
$$




$$
\pi_{s}=Q m_{s}+v_{s}
$$

donde $P$ y $Q$ son las cargas, $l_{s}$ y $m_{s}$ son las variables latentes, y $u_{s} \mathrm{y} v_{s}$ son los términos de error. El primer componente de mínimos cuadrados parciales se obtiene proyectando el producto combinado de la variable objeto de las predicciones y las mismas proyecciones, $\sum_{s} \pi_{s} x_{i, s}$, en la dirección de las predicciones.

\section{IV}

\section{Análisis de los resultados de la predicción}

En esta sección se presentan algunos de los resultados más importantes obtenidos durante el ejercicio de simulación de un programa de predicción en tiempo real, con un factor recursivo y estimación de parámetros. Las predicciones disponibles cubren el período comprendido entre enero de 2007 y diciembre de 2011 (60 meses). Se decidió comenzar la estimación con 36 valores anteriores a la muestra y se obtuvieron proyecciones con un período de antelación en forma recursiva a partir de entonces, de modo que en el ejercicio se utilizó una muestra de predicciones de 24 valores. Debido a que el primer componente principal representa el $89 \%$ de la variación y el segundo aumenta esta cantidad en solo 2 puntos porcentuales, se decidió utilizar solo un componente en los métodos de combinación.

Para fines comparativos, se emplearon también dos métodos comunes de combinación de predicciones: mínimos cuadrados ordinarios (MCO) y media corregida por sesgo (BC_mean) de las proyecciones en cada período $s$. La combinación de predicciones mediante MCO se realiza ajustando el modelo de regresión lineal múltiple

$$
\pi_{s}=c+\beta^{\prime} x_{s}+e_{s}
$$

donde los coeficientes estimados hallados con datos hasta el período $s$ se utilizaron para formar la verdadera combinación de proyecciones ex ante en $s+1$. En forma análoga, para obtener la media corregida por sesgo de las predicciones, se ajustó el modelo de regresión lineal simple

$$
\pi_{s}=c+\beta \overline{x_{s}}+e_{s}
$$

imponiendo $\beta=1$, donde $\overline{x_{s}}=\frac{1}{N} \sum_{i=1}^{N} x_{i, s}$ es la predicción media en el momento $s$. Los métodos de referencia para las comparaciones que figuran a continuación son la mediana y el promedio de las proyecciones.

Los resultados que se detallan en el cuadro 3 corresponden a las primeras predicciones mensuales con los datos faltantes imputados mediante uno de los dos métodos descritos anteriormente. ME denota el error medio de predicción que permite apreciar potenciales sesgos en el método de proyección, y RMSE corresponde a la raíz cuadrada del error cuadrático medio empleado como medida de precisión absoluta, pues se expresa en las mismas unidades de la tasa de inflación. Se emplea la U de Theil para establecer comparaciones de precisión relativa con respecto a la predicción promedio, que se toma como referencia por tratarse del método de combinación más sencillo. Se utiliza la precisión como medida principal para calificar las proyecciones y puede observarse que, con $N=18$, el segundo método de imputación es solo ligeramente mejor que el primero, de modo que el método de imputación no es en realidad tan importante. Dado que hay 5 expertos que no contestan a la encuesta en el $20 \%$ de las primeras predicciones mensuales y el $35 \%$ de las segundas, se hace un análisis basado en los 13 expertos restantes. En las últimas columnas del cuadro 3 se detallan los resultados relativos a $N=13$, donde se vuelve evidente que la reducción del número de analistas no afecta a las conclusiones que se pueden obtener con el segundo método de imputación y $N=18$.

A continuación, se presentan algunas conclusiones que surgen del cuadro 3. De acuerdo con los valores del error medio de predicción, no se aprecian sesgos importantes en ninguno de los métodos de combinación. De hecho, la relación señal-ruido $|\sqrt{24} \mathrm{ME} / \mathrm{RMSE}|$ similar a una estadística $|t|$ se sitúa en el rango $(0,77,1,40)$ para el primer método de imputación y $N=18$, a $(1,17,1,40)$ para el segundo método de imputación y $N=18$, y a $(0,98,1,40)$ para $N=13$. La mayor de estas razones se obtiene siempre con el promedio, sin importar el método de imputación o el número de expertos utilizados. Las estadísticas del error cuadrático medio y U de Theil 
Resultados de la primera predicción mensuala

\begin{tabular}{|c|c|c|c|c|c|c|c|c|c|}
\hline \multirow{3}{*}{$\begin{array}{l}\text { Expertos } \\
\text { Métodos de combinación }\end{array}$} & \multirow{2}{*}{\multicolumn{3}{|c|}{$\begin{array}{c}\text { Método de imputación } 1 \\
\qquad N^{\mathrm{b}}=18\end{array}$}} & \multicolumn{6}{|c|}{ Método de imputación 2} \\
\hline & & & & \multicolumn{3}{|c|}{$N^{\mathrm{b}}=18$} & \multicolumn{3}{|c|}{$N^{\mathrm{b}}=13$} \\
\hline & ME & RMSE & $\begin{array}{l}\text { U de } \\
\text { Theil }\end{array}$ & ME & RMSE & $\begin{array}{l}\text { U de } \\
\text { Theil }\end{array}$ & ME & RMSE & $\begin{array}{l}\text { U de } \\
\text { Theil }\end{array}$ \\
\hline Componentes principales & $-0,03$ & 0,19 & 0,80 & $-0,05$ & 0,18 & 0,73 & $-0,04$ & 0,18 & 0,75 \\
\hline Modelos de factores & $-0,04$ & 0,19 & 0,76 & $-0,05$ & 0,18 & 0,73 & $-0,05$ & 0,18 & 0,74 \\
\hline L1FM & $-0,03$ & 0,19 & 0,79 & $-0,05$ & 0,18 & 0,73 & $-0,04$ & 0,18 & 0,74 \\
\hline Mínimos cuadrados parciales & $-0,04$ & 0,19 & 0,78 & $-0,05$ & 0,18 & 0,72 & $-0,04$ & 0,18 & 0,75 \\
\hline $\mathrm{MCO}$ & $-0,05$ & 0,18 & 0,72 & 0,06 & 0,23 & 1,25 & 0,04 & 0,20 & 0,91 \\
\hline Media corregida por sesgo & $-0,04$ & 0,21 & 0,98 & 0,06 & 0,21 & 1,00 & $-0,04$ & 0,21 & 1,00 \\
\hline Mediana & $-0,05$ & 0,21 & 0,95 & $-0,05$ & 0,21 & 1,00 & $-0,05$ & 0,20 & 0,97 \\
\hline Promedio & $-0,06$ & 0,21 & - & $-0,06$ & 0,21 & - & $-0,06$ & 0,21 & - \\
\hline
\end{tabular}

Fuente: elaboración propia.

a Sobre la base de información hasta el mes anterior. Muestra de predicciones $=24$.

b $N=$ número de expertos considerados.

RMSE corresponde a la raíz cuadrada del error cuadrático medio.

ME denota el error medio de predicción.

MCO: mínimos cuadrados ordinarios.

revelan que los métodos de combinación (componentes principales, modelos de factores, L1FM y mínimos cuadrados parciales) son los más precisos al emplear el segundo método de imputación, mientras que el método de MCo lo es al utilizar el primero. En consecuencia, se decidió utilizar el método de combinación más sencillo, es decir, el análisis de componentes principales.

El gráfico 2, donde se pueden apreciar visualmente el desempeño de la predicción combinada y los correspondientes errores de proyección, se presenta como complemento del cuadro 3 . Se observa claramente que no hay patrones sistemáticos en los errores de predicción y que el comportamiento es similar ya sea que la inflación aumente o disminuya, aunque en apariencia la proyección combinada tiende a exagerar en los episodios más bajos y altos de inflación. El nivel y la varianza de los errores de predicción son razonablemente estables y no muestran pruebas de ineficacia.

$\mathrm{Al}$ igual que en el ejercicio anterior, en el cuadro $4 \mathrm{y}$ el gráfico 3 se proporciona un resumen de los resultados de la combinación de las segundas predicciones mensuales mediante los datos faltantes imputados con los métodos 1 y 2. En el cuadro 4 se revela que, en general, los errores medios de predicción son menores que los del cuadro 3 , de modo que, una vez más, ninguno de los métodos de combinación induce sesgos relevantes. En general, los valores relativos al error cuadrático medio también son más pequeños que los del cuadro 3 (a razón de alrededor de 11:18, excepto por el método de MCO cuyo desempeño es peor en comparación con los demás), de modo que las segundas predicciones mensuales combinadas son más precisas que las anteriores. Esto ocurre sobre todo con respecto a los métodos de combinación de promedio y mediana, mientras que el único método de combinación que supera al promedio es la mediana. Por otra parte, en el gráfico 3 se muestra claramente la proximidad entre la proyección combinada - la mediana - y la inflación observada. Incluso la exageración que era evidente en las primeras proyecciones mensuales combinadas disminuyó al combinar las segundas predicciones mensuales.

En resumen, sobre la base de los cuadros 3 y 4, se puede afirmar que la elección de un método de imputación es básicamente irrelevante al comparar los métodos de combinación. No obstante, se prefiere utilizar el segundo método porque permite ver las cosas con mayor claridad. Tampoco tiene sentido descartar los datos de los cinco expertos que realizaron menos predicciones que los otros, porque los resultados de los procedimientos de combinación son robustos a la presencia de esos expertos (desde luego con sus datos faltantes imputados con el segundo método de imputación). En cuanto a la elección de un método para combinar las proyecciones de los analistas, es evidente que hay un margen para mejorar con respecto al promedio. Por una parte, las primeras predicciones mensuales se combinan de la mejor manera mediante el análisis de componentes principales, que se elige porque es fácil de usar y proporciona una interpretación razonablemente sencilla de la combinación empleada. Por otra, las segundas proyecciones mensuales deberían combinarse con la mediana, lo que también constituye una técnica muy sencilla y fácil de usar. Estos resultados coinciden con las conclusiones que surgen de 
GRÁFICO 2

Primeras predicciones mensuales combinadas obtenidas mediante componentes principales

(En porcentajes)
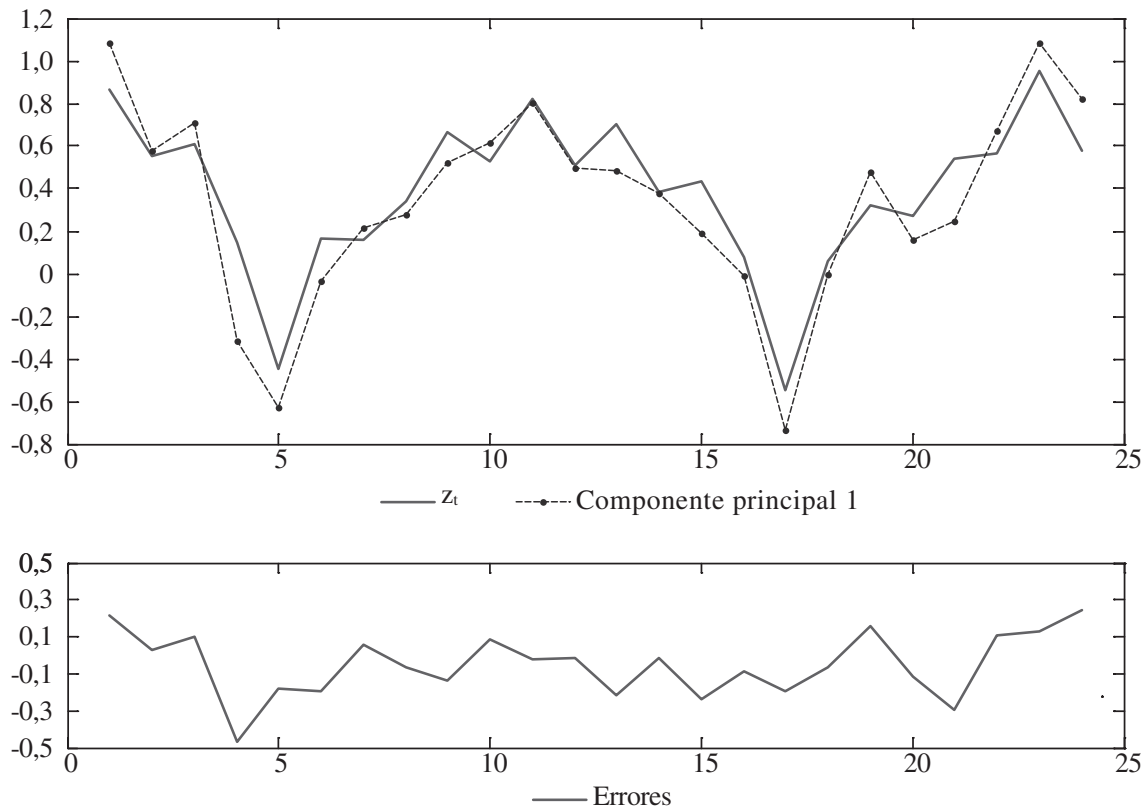

Fuente: elaboración propia.

CUADRO 4

Resultados de la segunda predicción mensuala

\begin{tabular}{|c|c|c|c|c|c|c|c|c|c|}
\hline \multirow{3}{*}{$\begin{array}{l}\text { Expertos } \\
\text { Métodos de combinación }\end{array}$} & \multirow{2}{*}{\multicolumn{3}{|c|}{$\begin{array}{c}\text { Método de imputación } 1 \\
\qquad N^{\mathrm{b}}=18\end{array}$}} & \multicolumn{6}{|c|}{ Método de imputación 2} \\
\hline & & & & \multicolumn{3}{|c|}{$N^{\mathrm{b}}=18$} & \multicolumn{3}{|c|}{$N^{\mathrm{b}}=13$} \\
\hline & ME & RMSE & $\begin{array}{l}\text { U de } \\
\text { Theil }\end{array}$ & ME & RMSE & $\begin{array}{l}\text { U de } \\
\text { Theil }\end{array}$ & ME & RMSE & $\begin{array}{l}\text { U de } \\
\text { Theil }\end{array}$ \\
\hline Componentes principales & $-0,01$ & 0,12 & 1,17 & $-0,01$ & 0,11 & 1,70 & $-0,01$ & 0,11 & 1,99 \\
\hline Modelos de factores & $-0,01$ & 0,11 & 0,99 & $-0,01$ & 0,10 & 1,45 & $-0,01$ & 0,10 & 1,92 \\
\hline L1FM & $-0,01$ & 0,12 & 1,17 & $-0,01$ & 0,11 & 1,72 & $-0,01$ & 0,11 & 2,01 \\
\hline Mínimos cuadrados parciales & $-0,01$ & 0,12 & 1,17 & $-0,01$ & 0,11 & 1,70 & $-0,01$ & 0,11 & 1,92 \\
\hline MCO & $-0,06$ & 0,15 & 1,85 & $-0,04$ & 0,17 & 3,98 & $-0,05$ & 0,16 & 4,31 \\
\hline Media corregida por sesgo & $-0,01$ & 0,11 & 1,02 & $-0,01$ & 0,09 & 1,01 & $-0,01$ & 0,08 & 1,00 \\
\hline Mediana & $-0,02$ & 0,10 & 0,78 & $-0,01$ & 0,07 & 0,67 & $-0,01$ & 0,07 & 0,89 \\
\hline Promedio & $-0,01$ & 0,11 & - & $-0,01$ & 0,09 & - & $-0,02$ & 0,08 & - \\
\hline
\end{tabular}

Fuente: elaboración propia.

a Sobre la base de información hasta la primera quincena del mes. Muestra de predicciones $=24$.

b $N=$ número de expertos considerados.

RMSE corresponde a la raíz cuadrada del error cuadrático medio.

ME denota el error medio de predicción.

MCO: mínimos cuadrados ordinarios. 
GRÁFICO 3

Segundas predicciones mensuales combinadas con la mediana (En porcentajes)
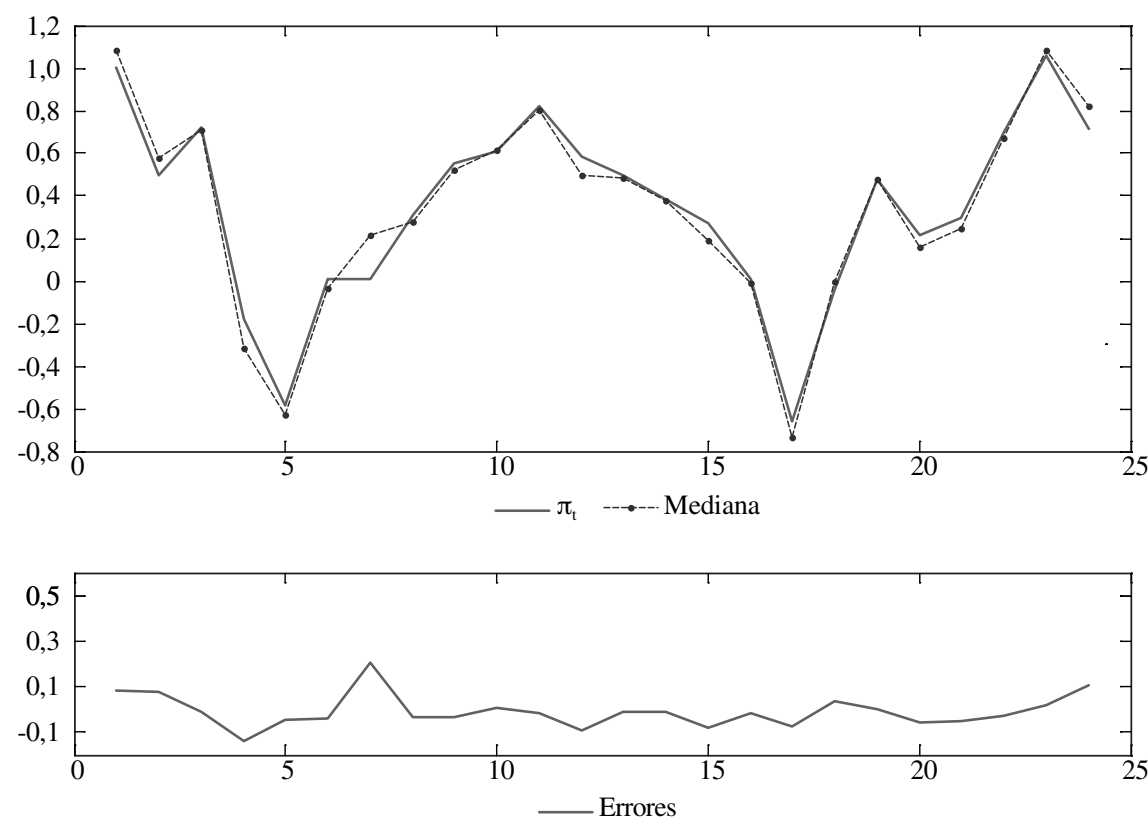

Fuente: elaboración propia.

la descomposición de panel detallada en el cuadro 2: en las muestras breves, cuando el aporte de las creencias comunes a la varianza de la predicción es mayor, los métodos sencillos son más adecuados que las técnicas de reducción de la dimensión multivariadas, porque no expresan la estimación de ningún parámetro. Por el contrario, cuando el aporte de las creencias comunes a la varianza del error de proyección es mayor, las técnicas de reducción de la dimensión parecen ser mejores que los métodos de referencia más sencillos.

Para complementar el análisis previo de precisión y sesgo de las predicciones, se pasa al estudio de la exactitud de la predicción. En el cuadro 5 se presentan las estadísticas relativas a la prueba de Diebold y Mariano (véase Diebold y Mariano, 1995) para la hipótesis nula de que cada método de combinación de predicciones empleado proporciona la misma exactitud predictiva que el promedio, utilizado como referencia. Cada estadística calculada debería compararse con una distribución normal estándar para declarar significación estadística. Los resultados de la prueba para el método de imputación son significativos al nivel del 5\% para todas las técnicas de reducción de la dimensión, con un signo negativo en la primera predicción mensual y un signo positivo en la segunda. Debido a que la diferencia corresponde a $\mathrm{d}=$ error cuadrático de proyección del método de combinación-error cuadrático de predicción del promedio, puede interpretarse que en el caso de la primera proyección esas técnicas resultan en un incremento estadísticamente significativo de la exactitud de la predicción con respecto al promedio, mientras que en el caso de la segunda ocurre lo contrario.

A la inversa, con el método de imputación 1 se aprecia que los grados de precisión significativamente diferentes ocurren solo con respecto a las primeras predicciones mensuales (al nivel del 5\%, excepto por los modelos de factores). Por otra parte, la exactitud de predicción de los otros métodos de combinación empleados no difiere de la del promedio, al nivel del 5\%, salvo en contadas ocasiones. En particular, para las segundas proyecciones mensuales, cuando el método empleado es MCO, este es considerablemente menos preciso que el promedio para ambos métodos de imputación. Por el contrario, la mediana es más precisa que el promedio. Una vez más, estos resultados proporcionan apoyo empírico al empleo de las técnicas de reducción para combinar las primeras predicciones mensuales y al uso de la mediana para las segundas proyecciones mensuales. 
CUADRO 5

Estadísticas de la prueba de Diebold-Mariano para igual exactitud de predicción de cada método de combinación en comparación con el promedioa

\begin{tabular}{|c|c|c|c|c|c|c|}
\hline \multirow{3}{*}{ Métodos de combinación } & \multicolumn{3}{|c|}{ Primeras predicciones mensuales } & \multicolumn{3}{|c|}{ Segundas predicciones mensuales } \\
\hline & \multirow{2}{*}{$\begin{array}{c}\text { Imputación } 1 \\
N^{\mathrm{b}}=18\end{array}$} & \multicolumn{2}{|c|}{ Imputación 2} & \multirow{2}{*}{$\begin{array}{l}\text { Imputación } 1 \\
\qquad N^{\mathrm{b}}=18\end{array}$} & \multicolumn{2}{|c|}{ Imputación 2} \\
\hline & & $N^{\mathrm{b}}=18$ & $N^{\mathrm{b}}=13$ & & $N^{\mathrm{b}}=18$ & $N^{\mathrm{b}}=13$ \\
\hline Componentes principales & $-2,26$ & $-2,29$ & $-2,24$ & 1,79 & 2,19 & 2,18 \\
\hline Modelos de factores & $-1,94$ & $-2,28$ & $-2,26$ & 1,79 & 2,13 & 2,16 \\
\hline L1FM & $-2,21$ & $-2,31$ & $-2,26$ & 1,80 & 2,20 & 2,20 \\
\hline Mínimos cuadrados parciales & $-2,25$ & $-2,32$ & $-2,28$ & 1,79 & 2,17 & 2,17 \\
\hline $\mathrm{MCO}$ & $-1,53$ & 0,34 & $-0,29$ & 2,48 & 2,66 & 3,02 \\
\hline Media corregida por sesgo & $-0,32$ & $-0,14$ & $-0,10$ & 0,92 & 0,73 & $-0,13$ \\
\hline Mediana & $-0,95$ & $-0,01$ & $-0,80$ & $-1,89$ & $-1,93$ & $-1,40$ \\
\hline
\end{tabular}

Fuente: elaboración propia.

${ }^{\text {a }}$ Muestra de predicciones $=24$.

b $N=$ número de expertos considerados.

MCO: mínimos cuadrados ordinarios.

\section{V}

\section{Conclusiones}

El principal objetivo de este estudio es mostrar que la información sobre las predicciones de inflación mensuales, proporcionada por la encuesta de analistas profesionales que realiza BANAMEX, puede aprovecharse mejor mediante técnicas de reducción de la dimensión y de combinación de las proyecciones. De hecho, se puso de manifiesto que dos de las técnicas más sencillas aquí utilizadas (componentes principales y mediana) produjeron mejores resultados que el promedio y, en consecuencia, superaron también las predicciones individuales de cada analista. Para establecer este hecho se tomó como referencia la predicción media (el promedio), pues generalmente es difícil de superar por otras técnicas de combinación más sofisticadas.

Si bien las primeras predicciones mensuales combinadas se consideran razonablemente precisas y libres de sesgos, las segundas son incluso mejores. Esto sugiere que los expertos realmente incorporan la información más reciente a sus segundas proyecciones. El hecho de que estas no requieran la aplicación de técnicas de reducción de la dimensión para obtener la predicción combinada, sino un simple cálculo de la mediana, corrobora lo anterior. Asimismo, al utilizar la mediana no es necesario estimar ningún vector de ponderación. La heterogeneidad entre los individuos en la primera encuesta podría explicar por qué la estimación de pesos óptimos produce mejores resultados de proyección que la asignación del mismo peso a todos los analistas, tratándolos como homogéneos. En la segunda encuesta, como se demostró mediante el análisis de panel, la homogeneidad entre los analistas es mayor. En este caso, tanto la muestra de proyecciones como la mediana superan a las técnicas de reducción de la dimensión. En esta segunda encuesta, en particular, los mejores resultados de predicción se obtuvieron con la mediana. 
ANEXO

\section{Predicción para la segunda quincena del mes}

Para obtener la predicción requerida se observa en primer lugar que

$$
\begin{aligned}
& \pi_{t}= \\
& 100\left[\frac{\left(q I P C_{2 t-1}+q I P C_{2 t}\right) / 2-\left(q I P C_{2(t-1)-1}+q I P C_{2(t-1)}\right) / 2}{\left(q I P C_{2(t-1)-1}+q I P C_{2(t-1)}\right) / 2}\right]
\end{aligned}
$$

de modo que

$$
\begin{aligned}
& p_{2 t}= \\
& \frac{\left(2 \pi_{t} I P C_{t-1}-2 p_{2 t-1} q I P C_{2(t-1)}-p_{2(t-1)} q I P C_{2(t-1)-1}\right)}{\left(q I P C_{2 t-1}\right)} .
\end{aligned}
$$

Entonces, por cada especialista $i=1, \ldots, N$, se pueden obtener predicciones para la segunda quincena del mes mediante

$$
\begin{aligned}
& y_{i, 2 t \mid 2 t-1}= \\
& \frac{\left(2 z_{i, t \mid 2 t-1} I P C_{t-1}-2 p_{2 t-1} q I P C_{2(t-1)}-p_{2(t-1)} q I P C_{2(t-1)-1}\right)}{\left(q I P C_{2 t-1}\right)}
\end{aligned}
$$

de manera que se puede obtener una serie temporal quincenal de proyecciones para la primera y la segunda quincenas de cada mes, es decir, tanto para $2 t-1=1,3$, .., $2 T-1$, como para $2 t=2,4, \ldots, 2 T$.
Aiolfi, M., C. Capistrán y A. Timmermann (2011), "Forecast combinations", The Oxford Handbook of Economic Forecasting, Michael Clements y David Hendry (eds.), Oxford, Oxford University Press.

Alonso, A. y otros (2011), "Seasonal dynamic factor analysis and bootstrap inference: application to electricity market forecasting", Technometrics, vol. 53, $\mathrm{N}^{\circ}$ 2, Taylor \& Francis.

Capistrán, C. y G. López-Moctezuma (2010a), "Las expectativas macroeconómicas de los especialistas: Una evaluación de pronósticos de corto plazo en México", El Trimestre Económico, vol. 77, № 306, México, D.F., Fondo de Cultura Económica.

(2010b), "Forecast revision of Mexican inflation and GDP growth", Working Papers, $\mathrm{N}^{\circ}$ 2010-11, México, D.F., Banco de México.

Bates, J.M. y C.W.J. Granger (1969), "The combination of forecasts", Operations Research Quarterly, vol. 20, ํㅜ 4, Birmingham, Operational Research Society.

Diebold, F.X. y R.S. Mariano (1995), "Comparing predictive accuracy", Journal of Business and Economic Statistics, vol. 13, $\mathrm{N}^{\circ}$ 3, Alexandria, American Statistical Association.

Friedman, M. (1976), Inflation and Unemployment. Nobel Memorial Lecture, Illinois, Universidad de Chicago, 13 de diciembre.

Ghysels, E., P. Santa-Clara y R. Valkanov (2004), "The MIDAS touch: mixed data sampling regression models", CIRANO Working Papers, $\mathrm{N}^{\circ}$ 2004s-20, Montreal, CIRANO.

Gómez, V. y A. Maravall (1996), "Programs tramo and seats: instructions for the user (with some updates)", Working Paper, $\mathrm{N}^{\circ} 9628$, Banco de España.

Lam, C., Q. Yao y N. Bathia (2011), "Estimation of latent factors for high-dimensional time series", Biometrika, vol. 98, $\mathrm{N}^{\circ} 4$, Oxford, Oxford University Press.
Lee, R.D. y L. Carter (1992), "Modeling and forecasting the time series of U.S. mortality", Journal of the American Statistical Association, vol. 87, $\mathrm{N}^{\circ}$ 419, Alexandria, American Statistical Association.

Newbold, P. y D.I. Harvey (2002), "Forecasting combination and encompassing", A Companion to Economic Forecasting, M. Clements y D. Hendry (eds.), Oxford, Blackwell.

Peña, D. y G.E.P. Box (1987), "Identifying a simplifying structure in time series", Journal of the American Statistical Association, vol. 82, $\mathrm{N}^{\circ} 399$, Alexandria, American Statistical Association.

Peña, D. y P. Poncela (2006), "Nonstationary dynamic factor analysis", Journal of Statistical Planning and Inference, vol. 136, $\mathrm{N}^{\circ} 4$, Amsterdam, Elsevier.

(2004), "Forecasting with nonstationary dynamic factor models", Journal of Econometrics, vol. 119, ํㅜ 2, Amsterdam, Elsevier.

Poncela, P. y E. Senra (2006), "A two factor model to forecast US inflation", Applied Economics, vol. 38, $\mathrm{N}^{\circ} 18$, Taylor \& Francis.

Poncela, P. y otros (2011), "Forecast combination through dimension reduction techniques", International Journal of Forecasting, vol. 27, $\mathrm{N}^{\circ}$ 2, Amsterdam, Elsevier.

Smith, J. y K.F. Wallis (2009), "A simple explanation of the forecast combination puzzle", Oxford Bulletin of Economics and Statistics, vol. 71, $\mathrm{N}^{\circ}$ 3, Oxford, Universidad de Oxford.

Timmermann, A. (2006), "Forecast combinations", Handbook of Economic Forecasting, G. Elliot, C.W.J. Granger y A. Timmerman (eds.), Amsterdam, Elsevier.

Woodford, M. (2003), Interest and Prices: Foundations of a Theory of Monetary Policy, Princeton, Princeton University Press. 